\title{
Hydroxytyrosol promotes autophagy by regulating SIRT1 against advanced oxidation protein product-induced NADPH oxidase and inflammatory response
}

\author{
TIAN SUN $^{1 *}$, QIAN CHEN ${ }^{2 *}$, SI-YUAN ZHU ${ }^{1}$, QIAN WU $^{1}$, CONG-RUI LIAO ${ }^{1}$, \\ ZHENG WANG $^{1}$, XIAO-HU WU ${ }^{3}$, HANG-TIAN WU ${ }^{3}$ and JIAN-TING CHEN ${ }^{1}$ \\ Departments of ${ }^{1}$ Orthopedic Spinal Surgery, ${ }^{2}$ Plastic Surgery and ${ }^{3}$ Orthopaedics and Traumatology, \\ Nanfang Hospital, Southern Medical University, Guangzhou, Guangdong 510515, P.R. China
}

Received December 12, 2018; Accepted July 8, 2019

DOI: $10.3892 /$ ijmm.2019.4300

\begin{abstract}
Advanced oxidation protein products (AOPPs) can trigger NADPH oxidase (NOX) and lead to the production of reactive oxygen species (ROS) in the pathophysiology of rheumatoid arthritis (RA). Hydroxytyrosol (HT) is a phenolic composite in olive oil that has antioxidant and anti-inflammatory effects and enhances autophagy. Early research has revealed that HT can activate the silent information regulator 1 (SIRT1) pathway to induce autophagy and alleviate the cartilage inflammatory response caused by $\mathrm{H}_{2} \mathrm{O}_{2}$. However, whether HT can attenuate AOPP-induced NOX and inflammatory responses remains to be elucidated. The present study aimed to investigate how HT can alleviate the damage caused by AOPPs. In cell experiments, chondrocytes were pre-stimulated with HT and then exposed to AOPPs. First, it was found that HT promoted autophagy through the SIRT1 pathway, increased the expression of autophagy-related proteins including microtubule-associated protein 1 light chain 3, autophagy related (ATG)5 and ATG7, and decreased the expression of P62. Furthermore, HT reduced the expression of NOX, which was affected by AOPPs in chondrocytes through the SIRT1 pathway. Finally, the expression of inflammatory cytokines caused by AOPPs was downregulated following HT treatment. In conclusion, it was found that HT reduced the expression of NOX and inhibited the inflammatory response caused by AOPPs in chondrocytes through the SIRT1 pathway.
\end{abstract}

Correspondence to: Professor Jian-Ting Chen, Department of Orthopedic Spinal Surgery, Nanfang Hospital, Southern Medical University, 1838 North Guangzhou Avenue, Guangzhou, Guangdong 510515, P.R. China

E-mail: chenjt99@tom.com

${ }^{*}$ Contributed equally

Key words: advanced oxidation protein products, NADPH oxidase, autophagy, inflammation, rat chondrocyte

\section{Introduction}

Rheumatoid arthritis (RA), a chronic progressive autoimmune disease, occurs primarily in the elderly and women. Its pathological features include long-term inflammation of synovial cells and chondrocytes leading to the destruction of bone and joints (1). In the pathogenesis of RA, tumor necrosis factor (TNF)- $\alpha$ and interleukin (IL)- 6 are the central pro-inflammatory factors able to induce the production of matrix metalloproteinases (MMPs) $(2,3)$. Studies have indicated that RA is associated with increased protein modification. Typical representatives are the production and accumulation of advanced oxidation protein products (AOPPs) in chondrocytes (4).

AOPPs are a class of protein cross-linking products containing a double tyrosine, which arise mainly from activated neutrophils during oxidative stress in vivo. Hypoxic acid (HCLO) generated by myeloperoxidase is formed by active proteins (mainly albumin), which is an oxidative stress protein marker and a general term for end products produced by protein oxidation (5). It has been found that the levels of AOPPs in plasma increase with age (6).

AOPPs have been recognized as a hallmark of oxidative stress and function as inflammatory mediators associated with the pathophysiology of several disorders, including accumulation in patients with arthritis $(7,8)$. Our previous studies confirmed that plasma AOPP levels were inversely correlated with age-related changes in bone mass in patients with RA and osteoporosis $(9,10)$. In addition, it was confirmed that AOPPs can accelerate cartilage degradation in rabbit arthritis models and apoptosis in murine chondrocytes $(4,11)$. NADPH oxidase (NOX), a membrane-bound enzyme composite, serves an essential function in the production of reactive oxygen species (ROS). AOPPs have been shown to induce apoptosis and inflammation of chondrocytes via NOX-dependent ROS production $(4,12)$. In general, AOPPs may represent a novel pathogenic factor accelerating the progression of RA $(4,12-14)$.

Hydroxytyrosol (HT), a polyphenol that mainly exists in extra virgin olive oil and red wine, possesses anti-inflammatory and antioxidant effects and is a suitable candidate for therapeutic interventions for arthritis $(15,16)$. Silent information 
regulator 1 (SIRT1), as a nicotinamide adenine dinucleotide (NAD+)-dependent histone deacetylase, regulates various physiological processes, including cell degeneration, cell survival and cell growth (17). Experiments have shown that HT can induce autophagy via the SIRT1 signaling pathway in primary chondrocytes of patients with osteoarthritis, and in human C-28/I2 chondrocytes for anti-oxidant and anti-inflammatory effects when challenged with $\mathrm{H}_{2} \mathrm{O}_{2}$ (18). However, whether and how HT regulates the autophagy of chondrocytes against AOPPs has not yet been investigated. Therefore, the present study aimed to investigate the role of HT in protection against AOPP-related RA by examining whether pharmacologically enhanced autophagy can prevent oxidative stress and inflammation-induced damage to chondrocytes.

\section{Materials and methods}

AOPP-rat serum albumin (RSA) preparation and determination. According to the method described previously, AOPP-RSA was prepared (10). The specific procedure was as follows: RSA solution $(20 \mathrm{mg} / \mathrm{ml}$, Sigma-Aldrich; Merck $\mathrm{KGaA}$ ) was exposed to $200 \mathrm{mmol} / \mathrm{l} \mathrm{HOCL}$ for $30 \mathrm{~min}$ at room temperature, following which the free HOCL was dialyzed off with phosphate-buffered saline (PBS) at $4^{\circ} \mathrm{C}$. Simultaneously, the control was incubated with only RSA dissolved in PBS. Endotoxins in all preparations were removed using a Detox-Gel column (Thermo Fisher Scientific, Inc.). The levels of endotoxin in the AOPP-RSA and unmodified RSA groups were assayed using an Amoebic Cell Lysate Assay kit (Sigma, Merck KGaA). All reagents prepared had an endotoxin concentration of $<0.025 \mathrm{EU} / \mathrm{ml}$. Finally, the determination of AOPP content was conducted as described in our previous study (19). The specific procedure was as follows: $200 \mu \mathrm{l}$ sample or chloramine-T was placed in each well of a 96-well plate, to which $20 \mu \mathrm{l}$ acetic acid was then added. The absorbance at $340 \mathrm{~nm}$ was immediately measured using a microplate reader. Finally, the AOPP content was determined to be 50.09 \pm 3.68 and $0.33 \pm 0.79 \mathrm{mmol} / \mathrm{g}$ protein in the AOPP-RSA and unmodified RSA groups, respectively.

Cell culture. Under sterile conditions, fresh cartilage from the knee joints of three newborn female Sprague-Dawley rats (weight, 8-10 g; Southern Medical University Animal Experient Center, Guangzhou, China) was isolated and washed with PBS. Animals were housed under standard conditions (temperature, $26-28^{\circ} \mathrm{C}$; relative humidity, $40-70 \%$ ), with a $12 \mathrm{~h}$ light/dark cycle and ad libitum access to food and water. The minced cartilage tissue was then digested with $0.25 \%$ Trypsin (Gibco, Thermo Fisher Scientific, Inc.) for $30 \mathrm{~min}$, followed by $0.2 \%$ collagenase II (Sigma, Merck KGaA) in DMEM//F12 (Gibco; Thermo Fisher Scientific, Inc.) for further digestion of the cartilage tissue $\left(3 \mathrm{~h}\right.$ at $\left.37^{\circ} \mathrm{C}\right)$. The digested chondrocytes were centrifuged at $1,000 \mathrm{x} \mathrm{g}$ at room temperature for $5 \mathrm{~min}$, and the resulting articular chondrocytes were resuspended in DMEM/F12 containing 10\% FBS (Gibco, Thermo Fisher Scientific, Inc.) and antibiotics $(100 \mathrm{IU} / \mathrm{ml}$ penicillin and $100 \mathrm{IU} / \mathrm{ml}$ streptomycin, Gibco, Thermo Fisher Scientific, Inc.). The cells were then seeded onto a culture dish at $37^{\circ} \mathrm{C}$ in a humidified atmosphere $\left(5 \% \mathrm{CO}_{2}, 95 \%\right.$ air). The chondrocytes were passaged at a ratio of $1: 3$, on reaching $\sim 80-90 \%$ confluency. Inverted phase contrast microscopy, Alcian blue staining and immunohistochemical staining with collagen II were used to identify the first passage chondrocytes. Only first passage chondrocytes were used in the present study, in order to avoid phenotype loss. The study was approved by the Institutional Animal Care and Use Committee of Southern Medical University (Guangzhou, China).

CCK-8 assay. The chondrocytes were seeded in a 96-well plate at $37^{\circ} \mathrm{C}(10,000$ cells/well $)$ and cultured overnight. Prior to treatment, the cells were extensively washed with PBS and cultured in DMEM/F12 without serum for $12 \mathrm{~h}$, and then incubated in the absence or presence of $200 \mu \mathrm{g} / \mathrm{ml} \mathrm{AOPPs}$ at $37^{\circ} \mathrm{C}$; $75 \mu \mathrm{M}$ HT (Cayman Chemical) was added for $30 \mathrm{~min}$ prior to stimulation with AOPPs. The autophagy inhibitor, chloroquine (50 $\mu \mathrm{m}$; Macklin Biochemical Co., Ltd.) was added to the cell medium for 30 min prior to other treatments. Control cells received an equal volume of DMEM/F12 + 10\% FBS. Subsequently, CCK-8 $(5 \mathrm{mg} / \mathrm{ml})$ in a volume equal to $10 \%$ of the culture volume was added to the cells and incubated for $2 \mathrm{~h}$ at $37^{\circ} \mathrm{C}$. The optical density (OD) was then measured at $450 \mathrm{~nm}$ using a microplate reader (Tecan Group, Ltd.).

Determination of ROS generation. The oxidation-sensitive fluorescent probe 2',7'-dichlorofluorescein diacetate (DCFH-DA; Sigma, Merck KGaA) was used to assess the levels of intracellular ROS generation. First, the chondrocytes were seeded at $70 \%$ ( $1 \times 10^{4}$ per well) confluence into a 96-well plate and then labeled with $10 \mu \mathrm{M}$ probe diluted in serum-free $\mathrm{DMEM} / \mathrm{F} 12$ for $30 \mathrm{~min}$ in the dark. To induce the production of ROS, the chondrocytes were pre-treated with, or without, $75 \mu \mathrm{M}$ HT for $30 \mathrm{~min}$ prior to stimulation with AOPPs for $2 \mathrm{~h}$. Subsequently, PBS was used to replace the medium, and fluorescence was measured using a SpectraMaxM5 Multimode Microplate Reader at excitation and emission wavelengths of 488 and $525 \mathrm{~nm}$, respectively. The experiment was performed in triplicate for each condition.

Western blotting. The cultured chondrocytes were harvested and lysed in radioimmunoprecipitation assay lysis buffer (Beyotime Institute of Biotechnology). The protein concentration was determined using a BCA Protein Assay kit (Thermo Fisher Scientific, Inc.). Total protein $(35 \mu \mathrm{g})$ was separated via SDS-polyacrylamide gel electrophoresis using 10-12\% acrylamide gels and then electrotransferred onto PVDF membranes (Pall Life Science). The membranes were then pre-incubated in blocking buffer (5\% RSA) for $60 \mathrm{~min}$ at room temperature and further incubated with primary antibodies overnight at $4^{\circ} \mathrm{C}$. Membranes were washed three times with TBST (TBS with Tween) and subsequently incubated with secondary antibodies for $1 \mathrm{~h}$ at room temperature. Samples were then washed three times with TBST prior to detection with chemiluminescence detection reagents (Merck KGaA). The following antibodies (Abs) were used: Rabbit anti-NOX4 mAb (1:1,000; cat. no. ab133303), rabbit anti-NOX2 mAb (1:5,000; cat. no. ab129068) and anti-TNFa (1:2,000; cat. no. ab66579) from Abcam; rabbit anti-LC3B mAb (1:1,000; cat. no. \#3868), rabbit anti-P62 mAb (1:1,000; cat. no. \#39786), rabbit anti-Sirt1 mAb (1:1,000; cat. no. \#9475) rabbit anti-ATG5 mAb (1:1,000; cat. no. \#12994) 
and rabbit anti-ATG7 $\mathrm{mAb}(1: 1,000$, \#8558) from Cell Signaling Technology, Inc.; and goat anti-mouse (1:2,000; cat. no. BA1050) and goat anti-rabbit (1:2,000; cat. no. BA1056) IgG-horseradish peroxidase (HRP) from Boster Biological Technology Co, Ltd. A rabbit anti-GAPDH pAb (1:2,500; Abcam; cat. no. ab9485) was used as a loading control. The integrated density of all protein bands were analyzed with ImageJ software V1.47 (National Institutes of Health).

Reverse transcription-quantitative PCR (RT-qPCR) analysis. According to the manufacturer's protocol, the treated and control chondrocytes were collected for the extraction of total RNA using TRIzol reagent (Takara Biotechnology Co., Ltd.). The RNA samples (260/280 ratio $>2.0)$ were quantified using a NanoDrop 1000 spectrophotometer (Thermo Fisher Scientific, Inc.). The total RNA (1 $\mu \mathrm{g})$ was used for RT using the PrimeScript ${ }^{\circledR}$ RT Reagent kit with gDNA Eraser (Takara Biotechnology Co., Ltd.). The resulting cDNA was PCR amplified using an SYBR ${ }^{\circledR}$ Premix Ex Taq ${ }^{\mathrm{TM}}$ II kit (Takara Biotechnology Co., Ltd.) with each specific primer. The sequences for the primers used are listed in Table I. The PCR procedure were performed on the Roche LightCycler 480 PCR system (Roche Diagnostics). The steps, according to the manufacturer's instructions were as follows: Initial enzyme activation at $95^{\circ} \mathrm{C}$ for $10 \mathrm{~min}$; followed by 45 cycles of $95^{\circ} \mathrm{C}$ for $10 \mathrm{sec}, 60^{\circ} \mathrm{C}$ for $20 \mathrm{sec}$ and $65^{\circ} \mathrm{C}$ for $30 \mathrm{sec}$. Following the amplification phase, a cooling step was performed at $40^{\circ} \mathrm{C}$ for $10 \mathrm{sec}$ (ramp rate of $1.5^{\circ} \mathrm{C} / \mathrm{sec}$ ). The housekeeping gene glyceraldehyde-3-phosphate dehydrogenase (GAPDH) was used for normalization. The relative expression (fold-change from control) was calculated using the $2^{-\Delta \Delta \mathrm{Cq}}$ method as previously described (20).

RNA interference. Rat small interfering RNA (CCTCAAGCC ATGTTCGATA), negative control siRNA (NC siRNA; cat. no. siN05815122147) and the ribo FECT ${ }^{\mathrm{TM}} \mathrm{CP}$ Transfection kit were chemically synthesized/obtained by/from Guangzhou Ribobio Co., Ltd. The chondrocytes in antibiotic-free growth medium were seeded into 6-well plates at a density of $20,000 / \mathrm{cm}$. When the cells reached $70 \%$ confluency, they were transfected with a stock concentration of $20 \mu \mathrm{M}$ synthesized siRNA targeting rat SIRT1 (forward, 5'-CCUCAAGCC AUGUUCGAUA-3'; reverse, 5'-UAUCGAACAUGGCUU GAGG-3'), according to the manufacturer's instructions. The cells were incubated for $24 \mathrm{~h}$ and then used in subsequent experiments. mRNA and protein expression were evaluated following stimulation.

Immunocytochemical detection of autophagosomes. Following stimulation, autophagy was detected in viable cells using an Autophagy Detection kit (cat. no. ab139484, Abcam) according to the manufacturer's protocol. The nuclei were stained with DAPI (blue) and autophagic vesicles were stained with FITC (green). For analysis, the cells were fixed by incubation with methanol for $20 \mathrm{~min}$ at $-20^{\circ} \mathrm{C}$. Staining was visualized using a laser scanning microscope (LSM 880 with Airyscan, Zeiss $\mathrm{GmbH}$ ) at excitation/emission wavelengths of 520/560 nm (FITC), and 360/460 nm (DAPI). The stained cells were counted in at least eight different fields of view at x400 magnification by two independent observers.
Table I. Sequences of primers used for reverse transcription-quantitative PCR analysis.

\begin{tabular}{lll}
\hline Gene & Primer & \multicolumn{1}{c}{ Sequence (5'-3') } \\
\hline SIRT1 & Forward & GCTCGCCTTGCTGTGGACTTC \\
& Reverse & GTGACACAGAGATGGCTGGAACTG \\
NOX2 & Forward & GCCATTCACACCATTGCACATCTG \\
& Reverse & CCAACCGAGTCACAGCCACATAC \\
NOX4 & Forward & ACAGTCCTGGCTTACCTTCG \\
& Reverse & TTCTGGGATCCTCATTCTGG \\
IL-6 & Forward & TGCTCTGGTCTTCTGGAGTTCCG \\
& Reverse & GTTGGATGGTCTTGGTCCTTAGCC \\
TNF- $\alpha$ & Forward & GTAGCAAACCACCAAGCGGA \\
& Reverse & ATGGGCTCATACCAGGGCTT \\
MMP-13 & Forward & CAAGATGTGGAGTGCCTGATGTGG \\
& Reverse & GCGTGTGCCAGAAGACCAGAAG \\
GAPDH & Forward & GGCACAGTCAAGGCTGAGAATG \\
& Reverse & ATGGTGGTGAAGACGCCAGTA
\end{tabular}

SIRT1, silent information regulator 1; NOX, NAPDH oxidase; IL-6, interleukin-6; TNF- $\alpha$, tumor necrosis factor- $\alpha$; IL-6, interleukin-6; MMP-13, matrix metalloproteinase-13.

Enzyme-linked immunosorbent assay (ELISA). The chondrocytes were seeded into 6-well culture plates in DMEM/F12 $+10 \%$ FBS and incubated for $24 \mathrm{~h}$. Following pre-treatment with HT, the cells were exposed to AOPPs for $24 \mathrm{~h}$. Following this, the cell supernatants were collected and centrifuged at $12,000 \mathrm{x} \mathrm{g}$ for $15 \mathrm{~min}$ at $4^{\circ} \mathrm{C}$. The TNF- $\alpha$ ELISA kit (MEIMIAN) and IL-6 ELISA kit (MEIMIAN) were then used to detect cytokine secretion into the supernatant. The OD values were determined at $450 \mathrm{~nm}$ using a spectrophotometric plate reader (Molecular Devices, LLC). All experiments were performed in triplicate.

Statistical analysis. Data are presented as the mean \pm SEM of three independent experiments. One-way ANOVA followed by Tukey's post hoc test was used to determine the differences between groups. $\mathrm{P}<0.05$ was considered to indicate a statistically significant difference. All statistical analyses were performed using GraphPad Prism version 6.0 (GraphPad Software, Inc.).

\section{Results}

Protective effect of HT on chondrocyte viability. The first aim of the present study was to evaluate the protective effect of HT on the viability of rat chondrocytes in the presence of AOPPs using a CCK-8 assay. The chondrocyte cultures were exposed to different concentrations of AOPPs for $24 \mathrm{~h}$ and the results revealed that AOPPs had a concentration-dependent adverse effect on cells; as the concentration of AOPPs increased, cell viability decreased (Fig. 1A). Furthermore, the chondrocytes were pre-treated with HT $(75 \mu \mathrm{m})$, which reversed the adverse effects of AOPPs on cell viability in a time-dependent manner (Fig. 1B). In addition, when the chondrocytes were incubated 
A
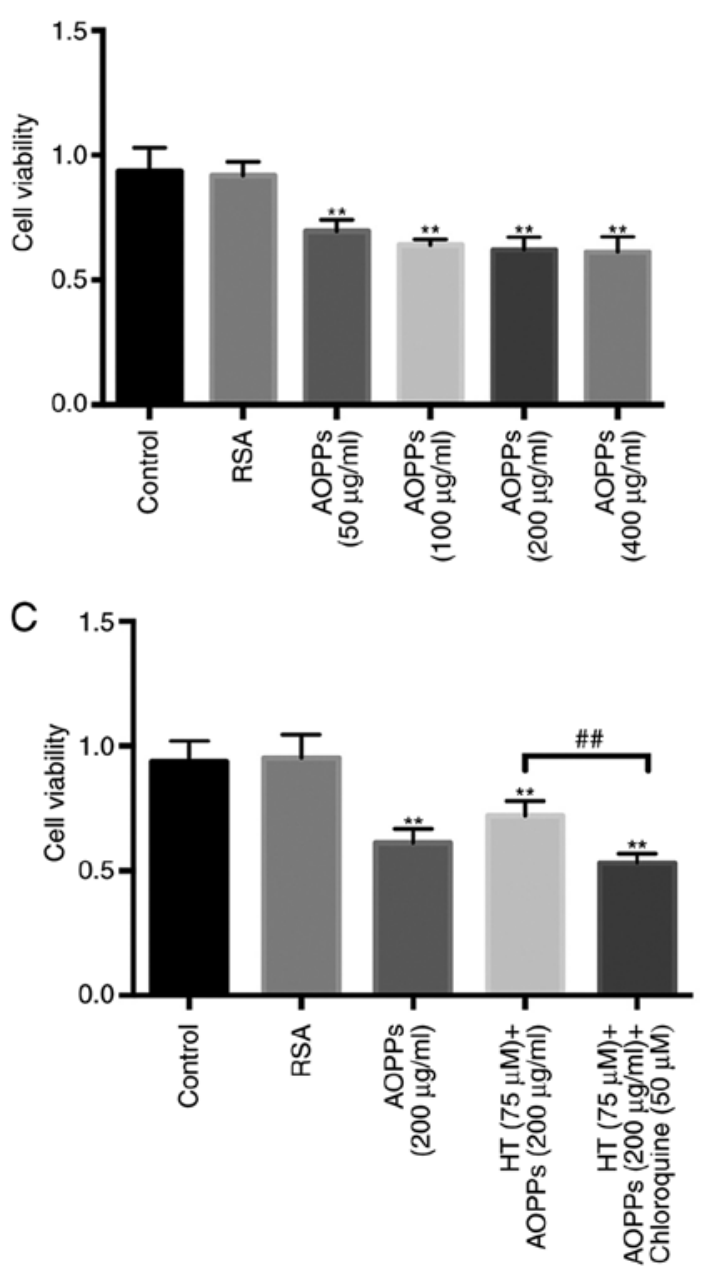

B
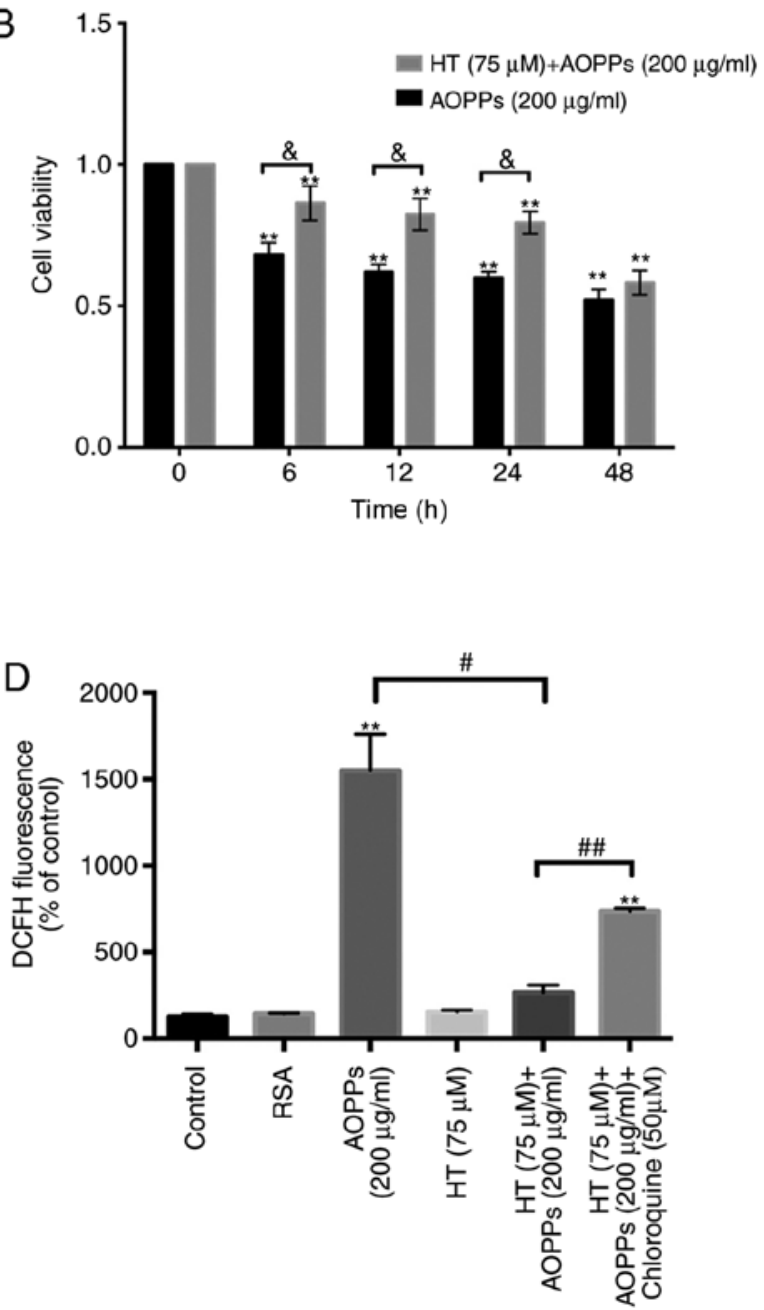

Figure 1. HT protects rat chondrocytes from AOPP-stimulated oxidative stress. Cell viability was determined using a CCK-8 assay. (A) Chondrocytes were stimulated with different concentrations of AOPPs for $24 \mathrm{~h}$. (B) Chondrocytes were pretreated with $75 \mu \mathrm{m} \mathrm{HT}$ for $30 \mathrm{~min}$ and then stimulated with $200 \mu \mathrm{g} / \mathrm{ml}$ AOPPs, or were treated with $200 \mu \mathrm{g} / \mathrm{ml}$ AOPPs only, for different durations. (C) Chondrocytes were pretreated with $75 \mu \mathrm{M}$ HT or $50 \mu \mathrm{M}$ chloroquine for $30 \mathrm{~min}$, and then exposed with $200 \mu \mathrm{g} / \mathrm{ml}$ AOPPs for $2 \mathrm{~h}$. (D) Fluorescent DCFH-DA was used to detect intracellular ROS levels. Data are expressed as the mean \pm SEM $(n=3) .{ }^{* *} \mathrm{P}<0.01$ vs. control group. ${ }^{\#} \mathrm{HT}+$ AOPPs group vs. AOPPs group. ${ }^{\# \#} \mathrm{P}<0.05 \mathrm{HT}+\mathrm{AOPPs}$ group vs. HT + AOPPs + chloroquine group. ${ }^{\&} \mathrm{P}<0.05 \mathrm{HT}+\mathrm{AOPPs}$ group vs. AOPPs group at 6,12 and $24 \mathrm{~h}$. HT, hydroxytyrosol; AOPPs, advanced oxidation protein products; RSA, rat serum albumin; DCFH-DA, 2',7'-dichlorofluorescein diacetate.

with the autophagy inhibitor chloroquine $(50 \mu \mathrm{m})$ for $30 \mathrm{~min}$ and then stimulated with HT $(75 \mu \mathrm{m})$ and AOPPs $(200 \mu \mathrm{g} / \mathrm{ml})$, the cell viability decreased (Fig. 1C).

$H T$ inhibits intracellular ROS generation stimulated by AOPPs. In our previous studies, it was demonstrated that NOX-dependent ROS generation was triggered by AOPPs. The consequences of these oxidative products include mitochondrial dysfunction and endoplasmic reticulum stress in chondrocytes. To determine whether HT can inhibit AOPP-induced ROS accumulation, intracellular ROS levels were evaluated following HT treatment. ROS production decreased in the HT-AOPP group compared with that in the AOPP only treatment group. To further verify the role of HT in ROS generation, intracellular ROS levels were also measured following the addition of chloroquine (an autophagy inhibitor). There was a significant increase in the generation of ROS in this group (Fig. 1D).

HT promotes autophagy in chondrocytes stimulated with AOPPs and upregulates the expression of SIRT1. When observing the autophagic process, LC3-II and P62 proteins are broadly utilized as markers of autophagy; reduced levels of P62 in combination with increased LC3-II are typically linked with autophagy (21). The bulk degradation of subcellular components leads to the production of autophagosomes/autolysosomes, marking the process of macroautophagy. Atg5 and Atg7 are considered essential genes in mammalian macroautophagy (22). To determine the optimal stimulation concentration and time required for HT, the present study evaluated different doses $(10,50$ and $75 \mu \mathrm{m})$ and times $(1,2,6,12$ and $24 \mathrm{~h})$. Exposure to $75 \mu \mathrm{m}$ HT caused the highest enhancement of autophagy proteins: LC3II increased from $1 \mathrm{~h}$ and reached a peak at $2 \mathrm{~h}$; P62 decreased, reaching the lowest level at $6 \mathrm{~h}$ (Fig. 2A and B). The chondrocytes were exposed to AOPPs $(200 \mu \mathrm{g} / \mathrm{ml})$ for $2 \mathrm{~h}$, in the presence or absence of HT $(75 \mu \mathrm{m})$. It was found that HT induced the protein expression of LC3-II and SIRT1 considerably. Furthermore, HT treatment decreased the protein level of P62 (Fig. 3A-C). Autophagosomes were assessed using an autophagy kit from Abcam. Cells actively 


\section{A}

A ATG-7

ATG-5

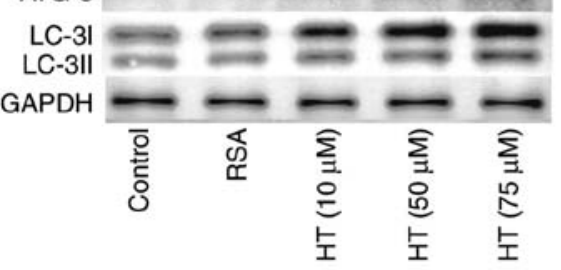

Control
RSA
HT $(10 \mu \mathrm{M})+$ AOPPs $(200 \mu \mathrm{g} / \mathrm{ml})$
HT $(50 \mu \mathrm{M})+$ AOPPs $(200 \mu \mathrm{g} / \mathrm{ml})$
HT $(75 \mu \mathrm{M})+$ AOPPs $(200 \mu \mathrm{g} / \mathrm{ml})$

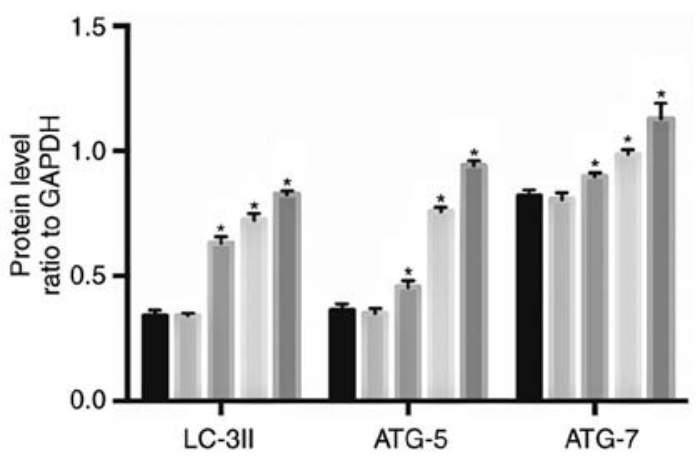

B
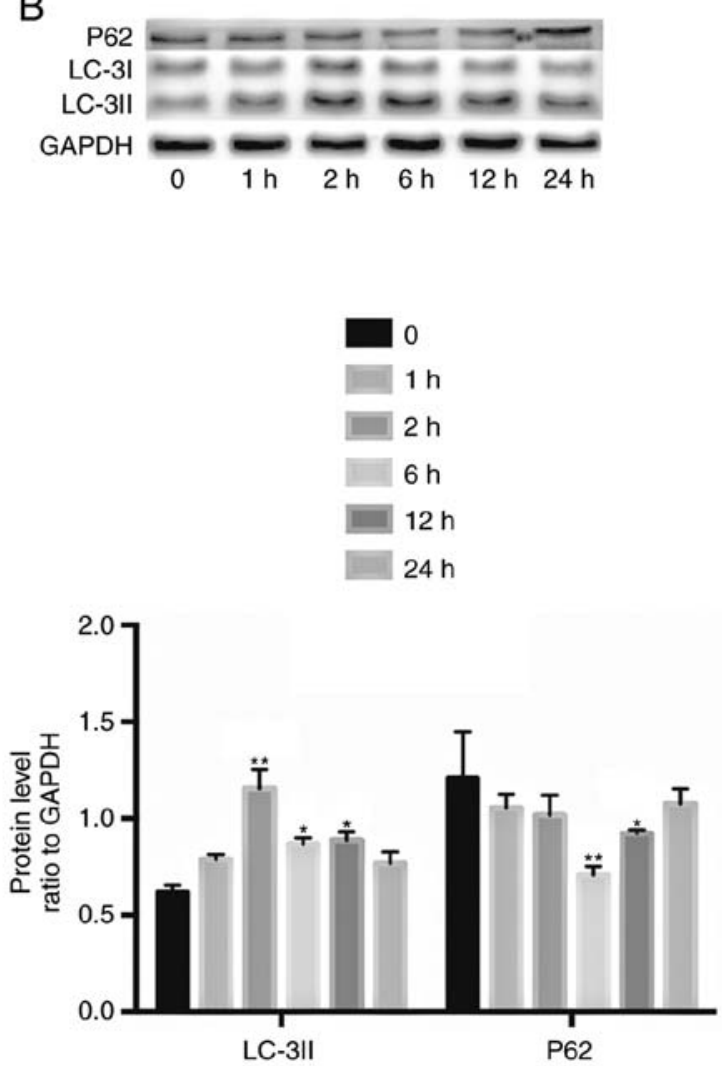

Figure 2. Autophagy-related cellular events following HT treatment. Autophagy-related proteins were detected by western blotting. (A) Autophagy proteins were enhanced by HT at different concentrations $(10,50$ and $75 \mu \mathrm{m})$ after $2 \mathrm{~h}$. (B) Chondrocytes were pretreated with $75 \mu \mathrm{m}$ HT for 30 min and then stimulated with $200 \mu \mathrm{g} / \mathrm{ml}$ AOPPs for different durations $(1,2,6,12$ and $24 \mathrm{~h})$. Data are expressed as the mean $\pm \mathrm{SEM}(\mathrm{n}=3)$. ${ }^{*}<0.05$ and ${ }^{* *} \mathrm{P}<0.01 \mathrm{vs}$. control group. HT, hydroxytyrosol; RSA, rat serum albumin; AOPPs, advanced oxidation protein products; LC-3II, microtubule-associated protein 1 light chain 3; ATG, autophagy related; GAPDH, glyceraldehyde-3-phosphate dehydrogenase.

undergoing autophagy exhibited a green fluorescent signal in the cytoplasm. Increased MDC fluorescence was observed in the presence of HT under AOPP stimulation, whereas the opposite effect was observed following treatment with AOPPs alone (Fig. 3D).

HT inhibits AOPP-induced NOX in chondrocytes. The protein expression of NOX was observed at various time points (1, 2, 4, 12 and $24 \mathrm{~h}$ ). The chondrocytes were exposed to AOPPs $(200 \mu \mathrm{g} / \mathrm{ml})$ in the presence or absence of HT $(75 \mu \mathrm{m})$. The results showed that the levels of NOX2 and NOX4 rose sharply by $1 \mathrm{~h}$, but decreased by $2 \mathrm{~h}$ (Fig. 4A). The possible effects of HT on NOX expression were also investigated. The protein expression levels of NOX 2 and NOX4 were markedly increased following AOPP stimulation. However, HT pretreatment led to a significant downregulation in the expression of NOX2 and NOX4 (Fig. 4B and C).

HT activates autophagy and inhibits AOPP-induced NOX in chondrocytes through the SIRT1 pathway. As our previous experiments showed, HT increases cell autophagy and upregulates SIRT1 pathway protein expression in chondrocytes under AOPP conditions. Subsequent experiments aimed to verify whether the function of HT in autophagy is influenced by the SIRT1 pathway. Chondrocytes transfected with SIRT1 siRNA were used for further experiments. Following stimulation subsequent to previous HT treatment, the expression levels of SIRT1 and LC3II were detected by western blotting and RT-qPCR analysis. The results indicated that the knockdown of SIRT1 decreased the protein expression of LC3II induced by HT (Fig. 5A and B). These findings suggest that HT increases autophagy under AOPP conditions by activating the SIRT1 pathway in chondrocytes.

To further clarify the roles of NOX2, NOX4 and SIRT1 in the protective effects of HT, chondrocytes transfected with SIRT1siRNA were used prior to HT-AOPP stimulation. The knockdown efficiency of SIRT1 was measured according to the mRNA expression of SIRT1 (Fig. 5C). The mRNA expression levels of NOX2 and NOX4 were assessed by RT-qPCR analysis. The results revealed that SIRT1 knockdown enhanced the mRNA expression of NOX2 and NOX4 in chondrocytes (Fig. 5D and E) These findings suggest that HT suppresses AOPP-induced NOX in chondrocytes by affecting the SIRT1 pathway.

HT decreases the secretion of IL-6, TNF- $\alpha$ and MMP-13 in chondrocytes stimulated with AOPPs. Finally, the present study assessed the ability of HT to protect rat chondrocytes against inflammation under AOPP conditions. Initially, the effects of HT on AOPP-induced IL-6, TNF- $\alpha$ and MMP-13 were examined using western blot analysis. The results revealed that the AOPP-driven release of IL-6, TNF- $\alpha$ and MMP-13 was decreased by HT (Fig. 6A and B). Consistently, the protective effects of HT on the expression of IL- 6 , TNF- $\alpha$ 
A
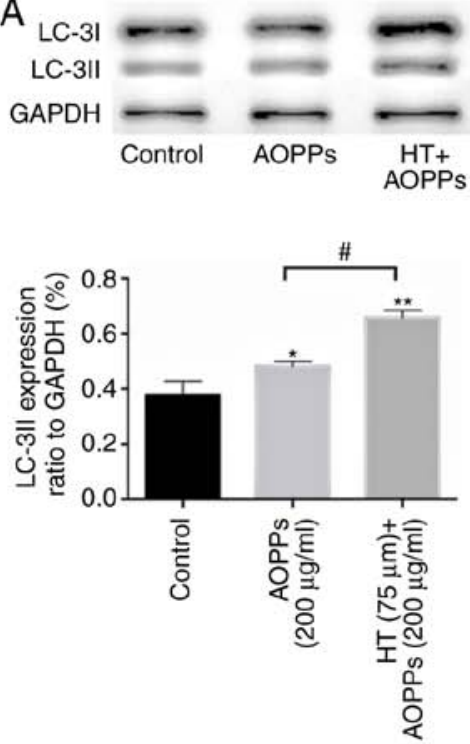

$\mathrm{D}$

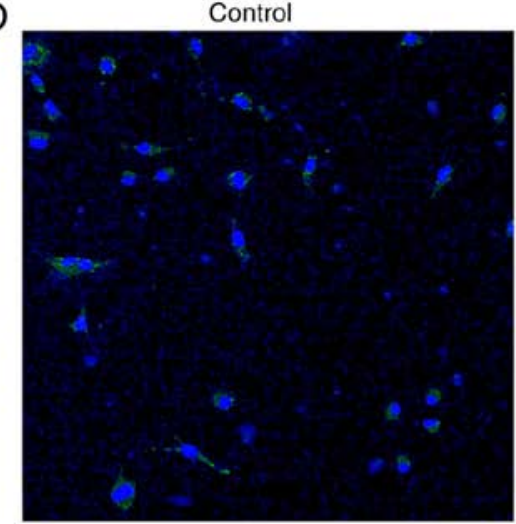

B
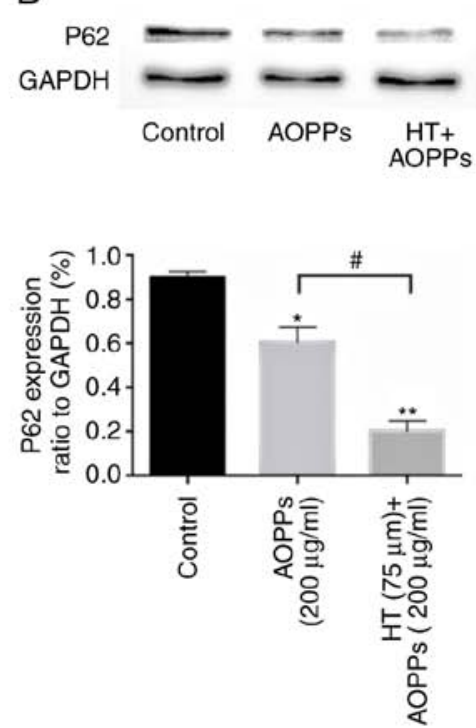

AOPPs

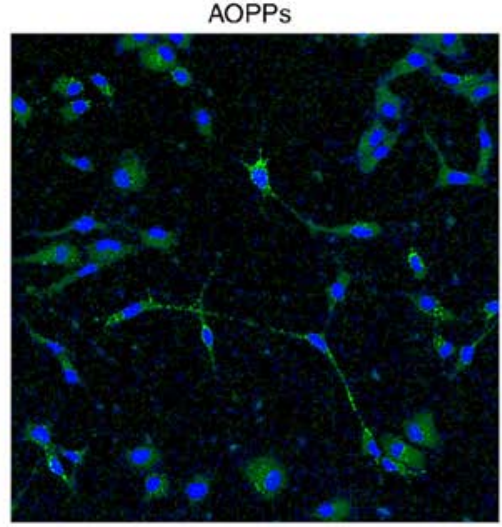

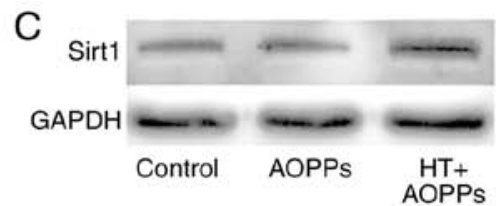
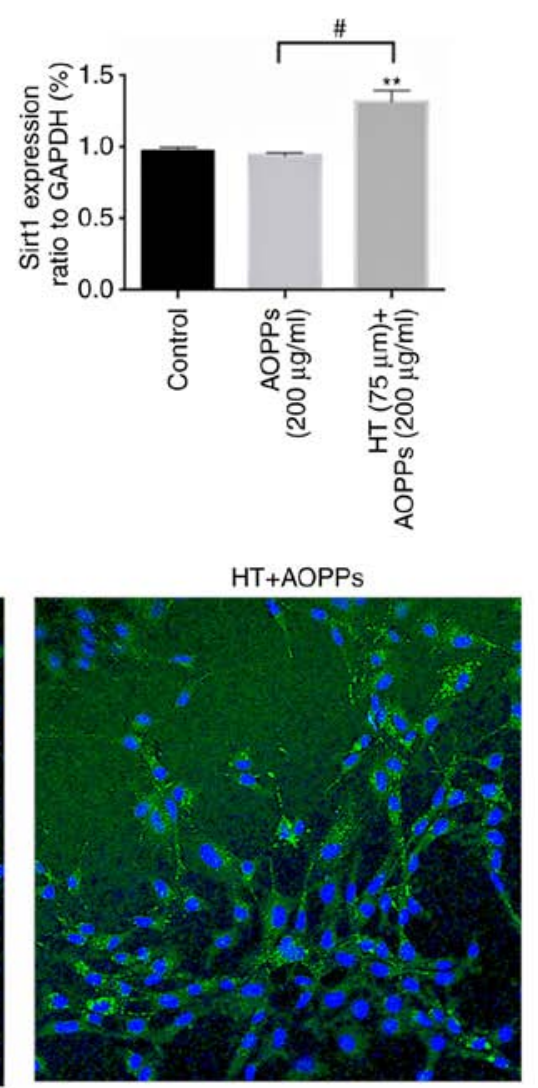

Figure 3. HT promotes autophagy and the SIRT1 pathway in chondrocytes stimulated with AOPPs. Chondrocytes were pretreated with $75 \mu \mathrm{M}$ HT for 30 min and then stimulated with AOPPs for $2 \mathrm{~h}$. Following incubation, the chondrocytes were collected for LC3II, P62 and GAPDH detection by western blotting. Relative quantification of the (A) LC3-II/GAPDH and (B) p62/GAPDH ratios is shown. (C) SIRT1 levels were also determined by western blotting, and relative quantification for the SIRT1/GAPDH ratio is shown. (D) A laser scanning microscope was used to visualize the formation of autophagy vacuoles (x60 magnification). Green indicates autophagy vacuoles; blue indicates nuclear staining. Data are expressed as the mean $\pm \mathrm{SEM}\left(\mathrm{n}=3\right.$ ). ${ }^{*} \mathrm{P}<0.05$ and ${ }^{* *} \mathrm{P}<0.01$ vs. control group. " $\mathrm{P}<0.05 \mathrm{HT}+\mathrm{AOPPs}$ group vs. AOPPs group. HT, hydroxytyrosol; AOPPs, advanced oxidation protein products; SIRT1, silent information regulator 1; LC-3II, microtubule-associated protein 1 light chain 3; ATG, autophagy related; GAPDH, glyceraldehyde-3-phosphate dehydrogenase.

and MMP-13 were quantified by RT-qPCR analysis. As shown in Fig. 6C-E, the mRNA levels of IL-6, TNF- $\alpha$ and MMP-13 in the HT treatment group were also reduced compared with those in the AOPP treatment group.

To further clarify the role of SIRT1 in HT cell protection, chondrocytes transfected with SIRT1 siRNA were used prior to HT-AOPP stimulation. The inflammatory cytokines IL-6 and TNF- $\alpha$ were detected via ELISAs. The results revealed that the knockdown of SIRT1 enhanced the levels of IL-6 and TNF- $\alpha$ inflammatory cytokines in chondrocytes prior to AOPP stimulation and HT co-treatment (Fig. 7A and B). Overall, the results indicated that HT protects chondrocytes from AOPP-induced inflammatory damage through the SIRT1 pathway.

\section{Discussion}

In the present study, the cellular and molecular events involved in the protective effect of HT on chondrocytes stimulated with AOPPs were investigated. The principal findings were as follows: i) HT protected chondrocytes against inflammation under AOPP conditions; ii) HT inhibited AOPP-induced NOX in chondrocytes stimulated with AOPPs through the SIRT1 pathway; iii) HT exerted protective effects on chondrocytes stimulated with AOPPs by promoting autophagy through the SIRT1 pathway.

The Mediterranean diet serves a significant role in complimentary pharmacotherapy for individuals living with RA and also contributes to reducing the risk of RA $(23,24)$. HT is a polyphenol, found mainly in extra virgin olive oil but also in red wine. It has a dominant antioxidant effect correlated with hydrogen donation and promotes radical stability. Furthermore, HT has other advantageous benefits to human health, specifically anti-inflammatory and anticancer effects, autophagy and mitochondrial function (25). MMPs can contribute to tissue remodeling during morphogenesis and inflammation; MMP secretion by chondrocytes has been observed in RA. Our previous study demonstrated that AOPPs can upregulate the mRNA and protein expression of TNF- $\alpha$, IL-1 $\beta$, MMP-3 and MMP-13 in a concentration-dependent 

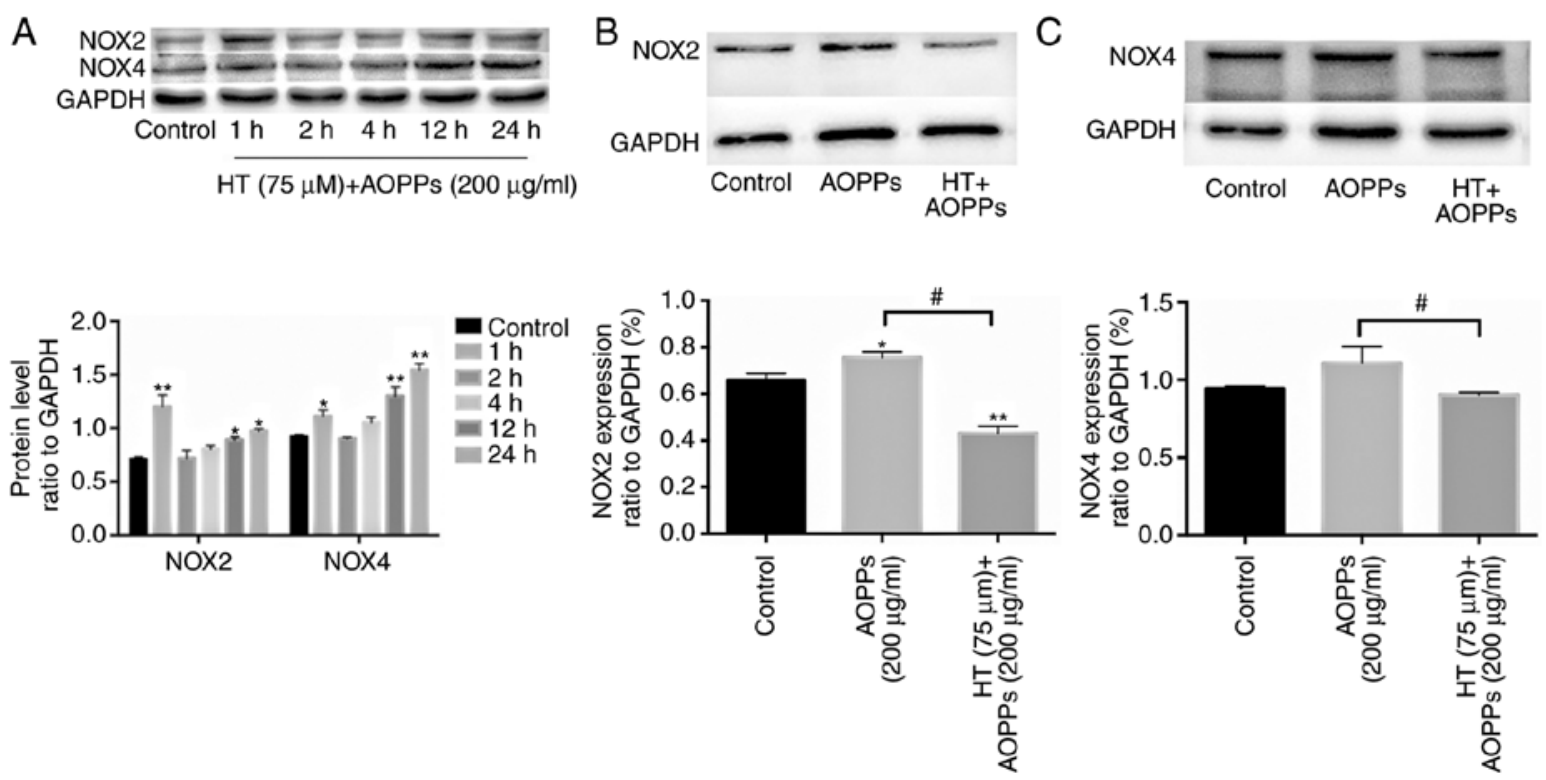

Figure 4. HT inhibits AOPP-induced NOX in chondrocytes. (A) Chondrocytes were pretreated with $75 \mu \mathrm{M}$ HT and then stimulated with AOPPs for different durations $(1,2,4,12$ and $24 \mathrm{~h}$ ). Chondrocytes were pretreated with or without $75 \mu \mathrm{M}$ HT for $30 \mathrm{~min}$, and then stimulated with AOPPs for $2 \mathrm{~h}$. The protein expression levels of (B) NOX2 and (C) NOX4 were detected by western blotting. Data are expressed as the mean \pm SEM $(\mathrm{n}=3)$. ${ }^{*} \mathrm{P}<0.05$ and ${ }^{* *} \mathrm{P}<0.01 \mathrm{vs}$. control group. ${ }^{\text {"P}} \mathrm{P}<0.05 \mathrm{HT}+\mathrm{AOPP}$ group vs. AOPPs group. HT, hydroxytyrosol; RSA, rat serum albumin; AOPPs, advanced oxidation protein products; NOX, NADPH oxidase; GAPDH, glyceraldehyde-3-phosphate dehydrogenase.

A
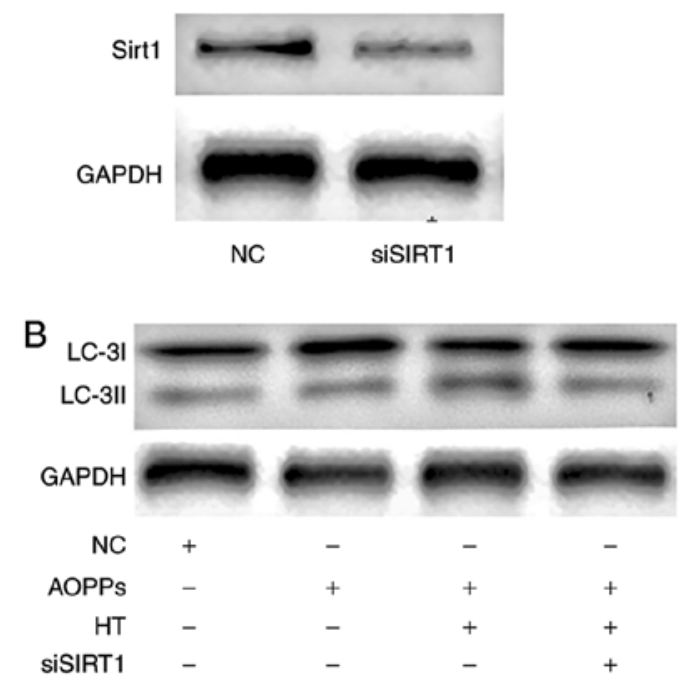
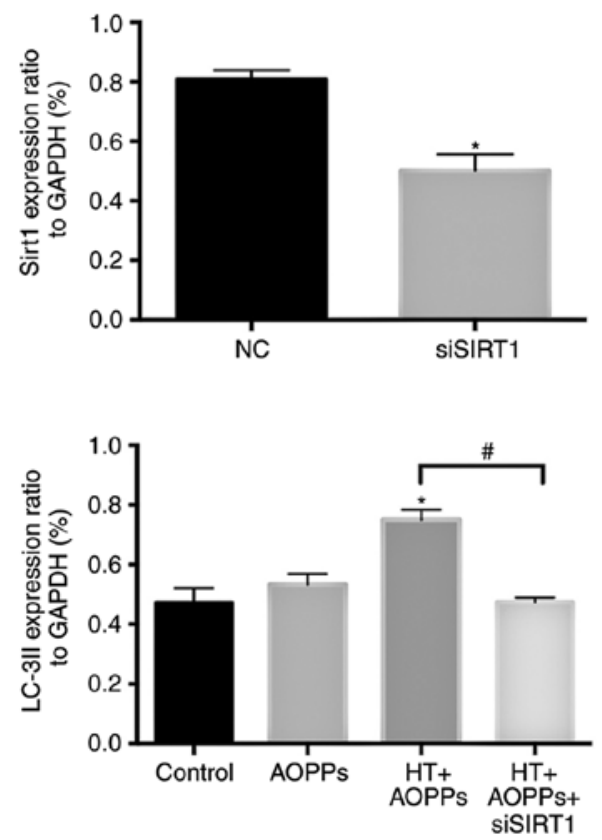
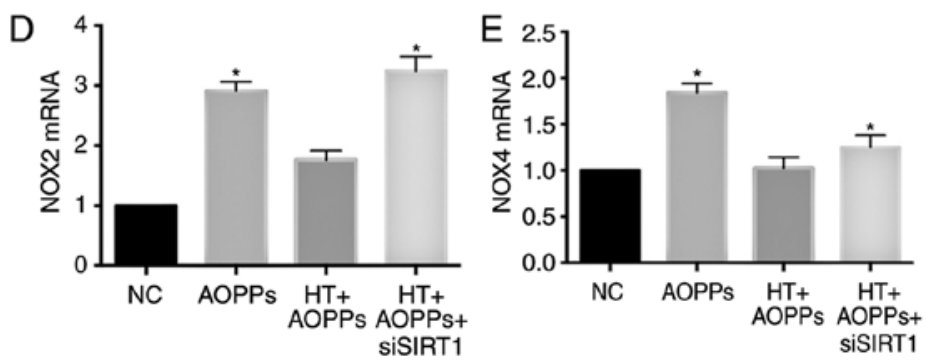

Figure 5. HT inhibits AOPP-induced NOX in chondrocytes through the SIRT1 pathway. Chondrocytes transfected with SIRT1 siRNA were pretreated with HT $(75 \mu \mathrm{M})$, and AOPPs were added for $2 \mathrm{~h}$. Protein expression levels of (A) SIRT1 and (B) LC3II were assessed by western blotting. Expression levels of (C) SIRT1, (D) NOX2 and (E) NOX4 were detected by reverse transcription-quantitative PCR analysis. The results are expressed as the mean \pm SEM (n=3). ${ }^{*} \mathrm{P}<0.05$ and ${ }^{* *} \mathrm{P}<0.01$ vs. control group. ${ }^{*} \mathrm{P}<0.05 \mathrm{HT}+\mathrm{AOPPs}$ group vs. HT + AOPPs + siSIRT1 group. HT, hydroxytyrosol; AOPPs, advanced oxidation protein products; LC-3II, microtubule-associated protein 1 light chain 3; NOX, NADPH oxidase; GAPDH, glyceraldehyde-3-phosphate dehydrogenase; SIRT1, silent information regulator 1; siSIRT1, small interfering RNA targeting SIRT1; NC, negative control. 
A

TNF-

GAPDH
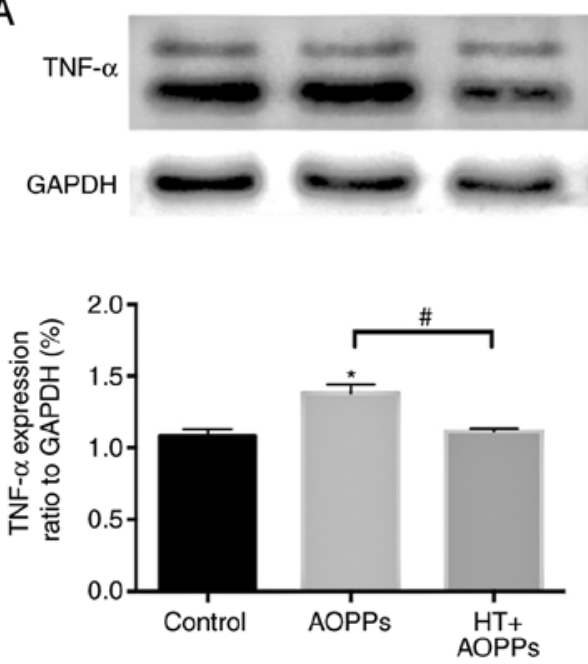

B
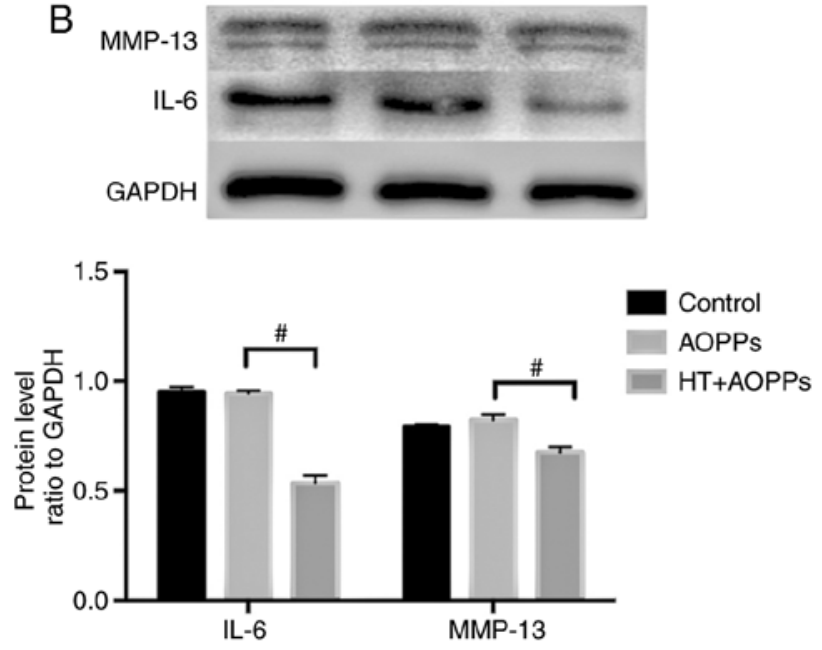
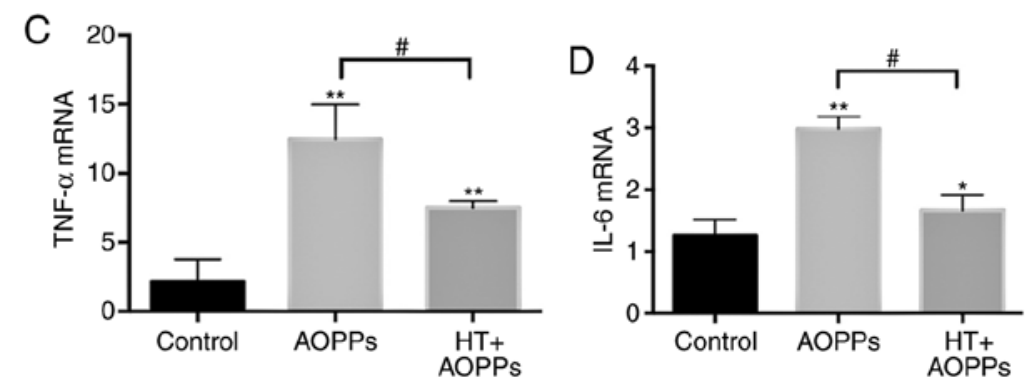

E

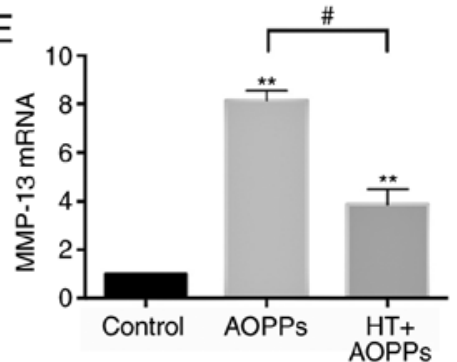

Figure 6. HT inhibits inflammatory damage in chondrocytes. Chondrocytes were pre-treated with or without $75 \mu \mathrm{M}$ HT for 30 min and then stimulated with AOPPs for $24 \mathrm{~h}$. The protein expression levels of (A) TNF- $\alpha$, (B) IL-6 and MMP-13 were detected by western blotting and mRNA levels of (C) TNF- $\alpha$, (D) IL-6 and (E) MMP-13 were detected by reverse transcription-quantitative PCR analysis. The results are expressed as the mean \pm SEM $(n=3)$. "P<0.05 and ${ }^{* *} \mathrm{P}<0.01$ vs. control group. ${ }^{\#} \mathrm{P}<0.05 \mathrm{HT}+$ AOPPs group vs. AOPPs group. HT, hydroxytyrosol; AOPPs, advanced oxidation protein products; TNF- $\alpha$, tumor necrosis factor- $\alpha$; IL-6, interleukin-6; MMP-13, matrix metalloproteinase-13; GAPDH, glyceraldehyde-3-phosphate dehydrogenase.
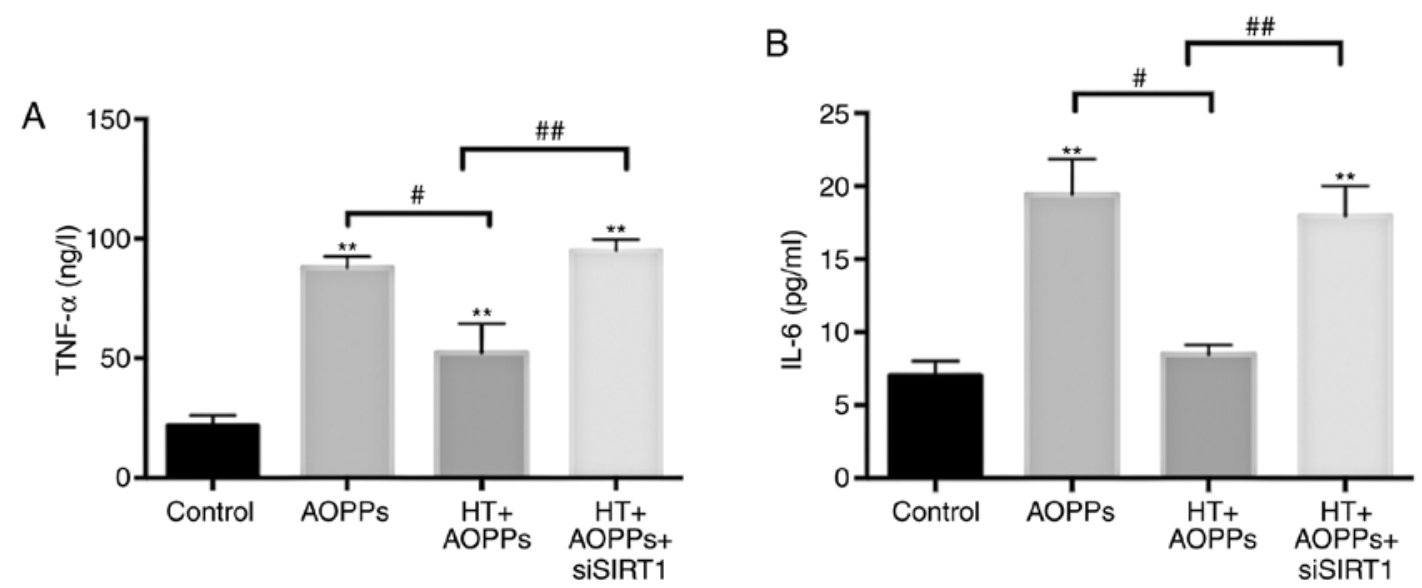

Figure 7. HT reduces the expression of (A) TNF- $\alpha$ and (B) IL-6 through the SIRT1 pathway. Chondrocytes transfected with SIRT1 siRNA were pretreated with HT $(75 \mu \mathrm{M})$ prior to stimulation with AOPPs for $2 \mathrm{~h}$. Enzyme-linked immunosorbent assays were used to detect the secretion of TNF- $\alpha$ and IL-6. The results are expressed as the mean $\pm \mathrm{SEM}(\mathrm{n}=3)$. ${ }^{* *} \mathrm{P}<0.01$ vs. control group. ${ }^{\#} \mathrm{P}<0.05 \mathrm{HT}+\mathrm{AOPPs}$ group vs. AOPPs group. ${ }^{\# \#} \mathrm{P}<0.05 \mathrm{HT}+\mathrm{AOPPs}$ group vs. HT + AOPPs + siSIRT1 group. HT, hydroxytyrosol; AOPPs, advanced oxidation protein products; SIRT1, SIRT1, silent information regulator 1; TNF- $\alpha$, tumor necrosis factor- $\alpha$; IL-6, interleukin-6; siRNA, small interfering RNA targeting SIRT1.

manner (11). However, the role of HT under AOPP conditions has not been investigated, to the best of our knowledge. The present study found that the treatment of chondrocytes with HT encouraged vital functional recovery; the expression levels of inflammatory TNF- $\alpha$ and IL-6, and the levels of MMP-13 were substantially inhibited, as shown by western blot and
RT-qPCR analyses. Overall, these findings indicated that HT has a significant effect against AOPP-induced inflammatory damage in chondrocytes.

Autophagy is a self-degrading process that is important for balancing energy sources at critical moments of development and nutritional stress responses (26). An important hallmark 
of RA is chondrocyte inflammation (27). In early research, it was reported that autophagy has a substantial influence on RA within the modulation of ROS, which promotes oxidative stress and results in the pathological processes of inflammation and apoptosis (28). As shown in Fig. 2, it was found in the present study that HT increased the autophagy in chondrocytes under AOPP conditions. The autophagy was concentration-dependent and reached a peak at $2 \mathrm{~h}$. However, when the autophagy inhibitor chloroquine was added, cell viability decreased and ROS generation increased (Fig. 1C and D). Therefore, it appears that HT activates autophagy to alleviate oxidative stress damage in chondrocytes. In preliminary experiments, treatments were performed with HT and chloroquine alone. However, no statistically significant differences with the control group were found. Therefore, the present study did not include HT and chloroquine groups, separately.

In mammals, NOX is the main source of ROS generation (29). Our previous pre-laboratory experiments demonstrated that AOPPs induced a time-dependent activation of NOX2 and NOX4 (13). Cetrullo et al (30) showed that HT prevented chondrocyte death and lowered ROS levels under oxidative stress by inducing autophagy. However, there is currently no information on the association between NOX and HT. In the present study, when the chondrocytes were pre-treated for $30 \mathrm{~min}$ with HT and then co-cultured with AOPPs, it was found that the levels of NOX2 and NOX4 increased sharply by $1 \mathrm{~h}$, but decreased by $2 \mathrm{~h}$ (Fig. 4A). Therefore, it was hypothesized that this phenomenon is associated with autophagy. The level of autophagy was insufficient to inhibit the intracellular ROS generation stimulated by AOPPs before $2 \mathrm{~h}$, so the levels of NOX2 and NOX4 increased. As shown in Fig. 2B, it was found that HT and AOPP co-culture increased autophagy in chondrocytes at 2-6 h, which in turn reduced the expression of NOX2 and NOX4. After $6 \mathrm{~h}$, the level of HT-activated autophagy gradually decreased over time, and the antioxidant capacity gradually decreased, which caused the increasing expression levels of NOX2 and NOX4. Subsequently, further experiments were designed and the observation point at $2 \mathrm{~h}$ was selected. Following pre-treatment of the chondrocytes with HT for $30 \mathrm{~min}$, AOPPs were added for $2 \mathrm{~h}$. It was found that the levels of NOX4 and NOX 2 were decreased in the HT pre-treatment group, indicating that HT can activate autophagy and reduce the NOX induced by AOPPs in chondrocytes.

SIRT1, as a protein deacetylase, is essential for chondrocyte survival and cartilage homeostasis (31). The SIRT1 pathway is also considered to be involved with therapeutic reagents for RA; for example, resveratrol is effective for the treatment of RA due to the overexpression of SIRT1 $(32,33)$. It has been reported that the activation of SIRT1 can increase the expression of superoxide dismutase 2 and regulate autophagy (34). Consistently, as shown in Fig. 3A-D, HT induced autophagy and enhanced the expression of SIRT1 in the present study. These findings confirm that HT enhances autophagy under AOPP conditions by activating the SIRT1 pathway in chondrocytes. SIRT1 is also involved in reducing the production of NOX, and it has been confirmed that the inhibition of SIRT1 is involved in the upregulation of NOX subunits (35). However, the functional role of SIRT1 in the associations among HT, AOPPs and autophagy have not been investigated. Chondrocytes transfected with SIRT1 siRNA were used for verification. The knockdown of SIRT1 reduced the autophagy protein LC3II (Fig. 5A-C) and increased the expression of NOX2 and NOX4 (Fig. 5D and E) in chondrocytes following HT pretreatment. In addition, higher levels of TNF- $\alpha$ and IL- 6 were observed in the SIRT1-knockdown group (Fig. 7A and B). These findings suggest that the SIRT1 pathway serves a notable role in enhancing autophagy and protecting chondrocytes against AOPP damage.

However, the present study only investigated at the in vitro level and not the in vivo level. A possible mechanism underlying the link between HT and AOPPs in chondrocytes is thus tentatively proposed. The present study provides further evidence demonstrating the role of HT in lowering ROS and NOX levels against AOPP-induced inflammatory responses.

In conclusion, the present study confirmed that HT enhances autophagy to protect against AOPP-induced inflammatory damage in chondrocytes through the SIRT1 signaling pathway. These findings provide novel insights into the links between HT, autophagy and NOX in chondrocytes. These results suggest a novel molecular mechanism for the protective role of HT and a possible clinical application of HT in RA.

\section{Acknowledgements}

Not applicable.

\section{Funding}

This study was supported by the National Natural Science Foundation of China (grant no. 81772395).

\section{Availability of data and materials}

All data generated or analyzed during this study are included in this published article.

\section{Authors' contributions}

TS and QC designed the study and conducted all experiments. SYZ assisted with western blotting. QW and CRL assisted with primary cell culture. XHW assisted with immunocytochemical imaging. HTW and ZW revised the manuscript and also helped with the extraction of primary cells. JTC supervised all experiments. All authors were involved in writing the manuscript. All authors read and approved the final manuscript.

\section{Ethics approval and consent to participate}

This study was approved by the Institutional Animal Care and Use Committee of Southern Medical University (Guangzhou, China).

\section{Patient consent for publication}

Not applicable.

\section{Competing interests}

The authors declare that they have no competing interests. 


\section{References}

1. Cheung PP, Dougados M, Andre V, Balandraud N, Chales G, Chary-Valckenaere I, Chatelus E, Dernis E, Gill G, Gilson M, et al: Improving agreement in assessment of synovitis in rheumatoid arthritis. Joint Bone Spine 80: 155-159, 2013.

2. Lavocat F, Osta B and Miossec P: Increased sensitivity of rheumatoid synoviocytes to Schnurri-3 expression in TNFand IL-17A induced osteoblastic differentiation. Bone 87: 89-96, 2016.

3. Choi YJ, Lee WS, Lee EG, Sung MS and Yoo WH: Sulforaphane inhibits IL-1 $\beta$-induced proliferation of rheumatoid arthritis synovial fibroblasts and the production of MMPs, COX-2, and PGE2 Inflammation 37: 1496-1503, 2014.

4. Wu Q, Zhong ZM, Zhu SY, Liao CR, Pan Y, Zeng JH, Zheng S, Ding RT, Lin QS, Ye Q, et al: Advanced oxidation protein products induce chondrocyte apoptosis via receptor for advanced glycation end products-mediated, redox-dependent intrinsic apoptosis pathway. Apoptosis 21: 36-50, 2016.

5. Witko-Sarsat V, Friedlander $M$, Capeillère-Blandin $C$, Nguyen-Khoa T, Nguyen AT, Zingraff J, Jungers $P$ and Descamps-Latscha B: Advanced oxidation protein products as a novel marker of oxidative stress in uremia. Kidney Int 49 : 1304-1313, 1996.

6. Komosinska-Vassev K, Olczyk P, Winsz-Szczotka K, KuznikTrocha K, Klimek K and Olczyk K: Age- and gender-related alteration in plasma advanced oxidation protein products (AOPP) and glycosaminoglycan (GAG) concentrations in physiological ageing. Clin Chem Lab Med 50: 557-563, 2012.

7. Kalousová M, Skrha J and Zima T: Advanced glycation end-products and advanced oxidation protein products in patients with diabetes mellitus. Physiol Res 51: 597-604, 2002.

8. García-González A, Gaxiola-Robles R and Zenteno-Savin T: Oxidative stress in patients with rheumatoid arthritis. Rev Invest Clin 67: 46-53, 2015.

9. Zhang YB, Zhong ZM, Hou G, Jiang H and Chen JT: Involvement of oxidative stress in age-related bone loss. J Surg Res 169: e37-e42, 2011.

10. Xie F, Sun S, Xu A, Zheng S, Xue M, Wu P, Zeng JH and Bai L: Advanced oxidation protein products induce intestine epithelial cell death through a redox-dependent, $\mathrm{c}$-jun $\mathrm{N}$-terminal kinase and poly (ADP-ribose) polymerase-1-mediated pathway. Cell Death Dis 5: e1006, 2014.

11. Yu H, Ye WB, Zhong ZM, Ding RT and Chen JT: Effect of advanced oxidation protein products on articular cartilage and synovium in a rabbit osteoarthritis model. Orthop Surg 7: 161-167, 2015.

12. Zheng S, Zhong ZM, Qin S, Chen GX, Wu Q, Zeng JH, Ye WB, Li W, Yuan K, Yao L and Chen JT: Advanced oxidation protein products induce inflammatory response in fibroblast-like synoviocytes through NADPH oxidase-dependent activation of NF- $\kappa$ B. Cell Physiol Biochem 32: 972-985, 2013.

13. Ye W, Zhong Z, Zhu S, Zheng S, Xiao J, Song S, Yu H, Wu Q, Lin $Z$ and Chen $J$ : Advanced oxidation protein products induce catabolic effect through oxidant-dependent activation of NF- $\kappa \mathrm{B}$ pathway in human chondrocyte. Int Immunopharmacol 39: 149-157, 2016

14. Ye W, Zhu S, Liao C, Xiao J, Wu Q, Lin Z and Chen J: Advanced oxidation protein products induce apoptosis of human chondrocyte through reactive oxygen species-mediated mitochondrial dysfunction and endoplasmic reticulum stress pathways. Fundam Clin Pharmacol 31: 64-74, 2017.

15. Ghanbari R, Anwar F, Alkharfy KM, Gilani AH and Saari N: Valuable nutrients and functional bioactives in different parts of olive (Olea europaea L.)-a review. Int J Mol Sci 13: 3291-3340, 2012.

16. Chin KY and Pang KL: Therapeutic effects of olive and its derivatives on osteoarthritis: From bench to bedside. Nutrients 9: 2017.
17. Cerutti R, Pirinen E, Lamperti C, Marchet S, Sauve AA, Li W, Leoni V, Schon EA, Dantzer F, Auwerx J, et al: NAD(+)dependent activation of Sirtl corrects the phenotype in a mouse model of mitochondrial disease. Cell Metab 19: 1042-1049, 2014.

18. D'Adamo S, Cetrullo S, Guidotti S, Borzi RM and Flamigni F: Hydroxytyrosol modulates the levels of microRNA-9 and its target sirtuin-1 thereby counteracting oxidative stress-induced chondrocyte death. Osteoarthritis Cartilage 25: 600-610, 2017.

19. Wu Q, Zhong ZM, Pan Y, Zeng JH, Zheng S, Zhu SY and Chen JT: Advanced oxidation protein products as a novel marker of oxidative stress in postmenopausal osteoporosis. Med Sci Monit 21: 2428-2432, 2015.

20. Pfaffl MW: A new mathematical model for relative quantification in real-time RT-PCR. Nucleic Acids Res 29: e45, 2001.

21. Komatsu M and Ichimura Y: Physiological significance of selective degradation of $\mathrm{p} 62$ by autophagy. FEBS Lett 584 : 1374-1378, 2010.

22. Nishida Y, Arakawa S, Fujitani K, Yamaguchi H, Mizuta T, Kanaseki T, Komatsu M, Otsu K, Tsujimoto Y and Shimizu S: Corrigendum: Discovery of Atg5/Atg7-independent alternative macroautophagy. Nature 533: 130, 2016.

23. Forsyth C, Kouvari M, D'Cunha NM, Georgousopoulou EN, Panagiotakos DB, Mellor DD, Kellett J and Naumovski N: The effects of the Mediterranean diet on rheumatoid arthritis prevention and treatment: A systematic review of human prospective studies. Rheumatol Int 38: 737-747, 2018.

24. Philippou E and Nikiphorou E: Are we really what we eat? Nutrition and its role in the onset of rheumatoid arthritis. Autoimmun Rev 17: 1074-1077, 2018.

25. EcheverríaF,OrtizM,ValenzuelaR and Videla LA:Hydroxytyrosol and cytoprotection: A projection for clinical interventions. Int J Mol Sci 18: 2017.

26. Glick D, Barth S and Macleod KF: Autophagy: Cellular and molecular mechanisms. J Pathol 221: 3-12, 2010.

27. Harre U and Schett G: Cellular and molecular pathways of structural damage in rheumatoid arthritis. Semin Immunopathol 39: 355-363, 2017.

28. Vomero M, Barbati C, Colasanti T, Perricone C, Novelli L, Ceccarelli F, Spinelli FR, Di Franco M, Conti F, Valesini G and Alessandri C: Autophagy and rheumatoid arthritis: Current knowledges and future perspectives. Front Immunol 9: 1577, 2018.

29. Cai H and Harrison DG: Endothelial dysfunction in cardiovascular diseases: The role of oxidant stress. Circ Res 87: 840-844, 2000.

30. Cetrullo S, D'Adamo S, Guidotti S, Borzi RM and Flamigni F: Hydroxytyrosol prevents chondrocyte death under oxidative stress by inducing autophagy through sirtuin 1-dependent and -independent mechanisms. Biochim Biophys Acta 1860: 1181-1191, 2016.

31. Gabay O and Sanchez C: Epigenetics, sirtuins and osteoarthritis. Joint Bone Spine 79: 570-573, 2012.

32. Kong S, Yeung P and Fang D: The class III histone deacetylase sirtuin 1 in immune suppression and its therapeutic potential in rheumatoid arthritis. J Genet Genomics 40: 347-354, 2013.

33. Nakayama H, Yaguchi T, Yoshiya S and Nishizaki T: Resveratrol induces apoptosis MH7A human rheumatoid arthritis synovial cells in a sirtuin 1-dependent manner. Rheumatol Int 32: 151-157, 2012.

34. Lee IH, Cao L, Mostoslavsky R, Lombard DB, Liu J, Bruns NE, Tsokos M, Alt FW and Finkel T: A role for the NAD-dependent deacetylase Sirtl in the regulation of autophagy. Proc Natl Acad Sci USA 105: 3374-3379, 2008.

35. Wosniak J Jr, Santos CX, Kowaltowski AJ and Laurindo FR: Cross-talk between mitochondria and NADPH oxidase: Effects of mild mitochondrial dysfunction on angiotensin II-mediated increase in Nox isoform expression and activity in vascular smooth muscle cells. Antioxid Redox Signal 11: 1265-1278, 2009. 